\title{
INTEGRATED PHOTONICS
}

\section{Compact multiplexing}

Spatial multiplexers (SMUXs) for mode division multiplexing often involve multiple strategies for mode-selective excitation and the minimization of insertion and other losses. Haoshuo Chen, Roy van Uden, Chigo Okonkwo and Ton Koonen, working at the COBRA Institute at the Eindhoven University of Technology in The Netherlands, have reported a SMUX for mode division multiplexing that packages multiple strategies into compact components with a small footprint (Opt. Express 22, 31582-31594; 2014). They demonstrated photonic integrated SMUXs on both silicon-on-insulator (SOI) and indium phosphide ( $\mathrm{InP}$ ) platforms for selectively exciting modes.

The use of two-dimensional topcoupling provided the coupling interface between a few-mode fibre and the photonic integrated SMUX. A 3-spot SMUX realized using the InP platform with $45^{\circ}$ vertical mirrors and laser-inscribed waveguide technology was demonstrated as an option for a fully-packaged dualchannel 6-mode SMUX including two 6-core photonic lantern structures serving as a mode multiplexer and a

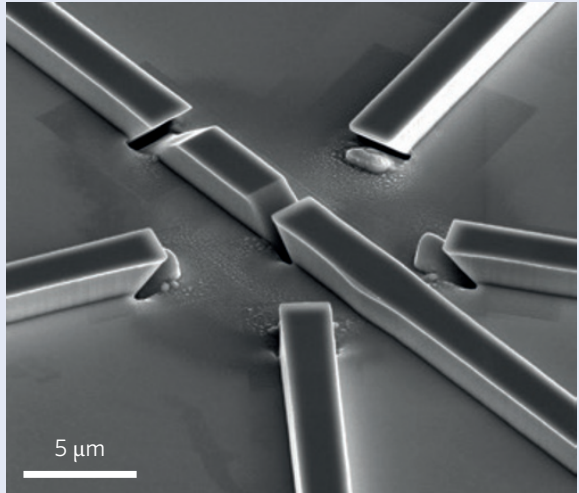

"Previous spatial multiplexers were mostly based on bulky free-space optics with large footprints, which can be challenging to scale up to support more spatial-mode channels," Chen explained. "Integrated photonic SMUXs can be incorporated with other functionality on the same chip, which is cost- and energy-efficient."

As always, there were some hurdles for the authors. Chen noted that the insertion losses of the experimentally fabricated vertical emitters can be greater than those predicted by the design. However, Chen said that future work will focus on optimizing designs and reducing insertion losses. The team would also like to integrate other functionality with the SMUX such as lasers, optical modulators for spatial division multiplexing transmitters, and create hybrids - photodetectors for spatial division multiplexing coherent receivers. The photonic SMUXs can be mass-manufactured and packaged for high reliability. dimensional top-coupling between fewmode spatial division multiplexing fibres and photonic integrated components.

\section{The rise of the GeSn laser}

The development of a group IV semiconductor laser that is CMOS-compatible represents a step towards the creation of fully integrated electronic and photonic circuitry.

\section{Kevin P. Homewood and Manon A. Lourenço}

$\mathrm{S}$ ilicon underpins the whole digital world. Sadly this 'champion' semiconductor for electronics lacks the key photonic capability of efficient light emission. As a result, the realization of a practical monolithic silicon laser that is electrically-driven and operates at roomtemperature has proved problematic, despite ongoing efforts and the achievement of lowtemperature lasing or gain ${ }^{1,2}$.

Currently, the only workable solution to the problem is a hybrid approach, where a conventional semiconductor laser system based on III-V materials, such as InP or GaAs, is wafer-bonded to a silicon-oninsulator (SOI) wafer. This approach has been pursued by Intel, the University of California and others with notable success ${ }^{3,4}$. However, it has the disadvantage of not offering the benefits and cost advantages inherent in monolithic silicon integration and CMOS fabrication technology.

As such, although there has been much progress in making silicon photonic devices ${ }^{5,6}$, the Holy Grail is still the development of electrically pumped lasers and optical amplifiers in silicon itself. Although this goal remains elusive, European scientists have now demonstrated the next best thing - a GeSnon-silicon laser that is CMOS-compatible. Writing in Nature Photonics, S. Wirths and co-workers ${ }^{7}$ report the first demonstration of lasing using a direct-gap group IV system grown on standard silicon substrates. Their approach using a germanium/tin alloy is of great interest as a true direct-bandgap semiconductor can be formed, which should enable an essentially conventional injection laser to be developed as part of a CMOS process.

Germanium, like silicon, is an indirectbandgap semiconductor and so is fundamentally a poor light emitter. However, unlike in silicon where the direct gap is far above the indirect-band edge $(\sim 3 \mathrm{eV})$, in germanium the direct gap is located nearby $(\sim 140 \mathrm{meV})$. Previously, tensile strain was applied to germanium to lower the direct gap 